\title{
A Mathematical Model for the Industrial Hazardous Waste Location-Routing Problem
}

\author{
Omid Boyer, ${ }^{1}$ Tang Sai Hong, ${ }^{2}$ Ali Pedram, ${ }^{2}$ \\ Rosnah Bt Mohd Yusuff, ${ }^{2}$ and Norzima Zulkifli ${ }^{2}$ \\ ${ }^{1}$ Department of Industrial Engineering, Islamic Azad University of Najafabad, Najafabad, Isfahan, Iran \\ ${ }^{2}$ Department of Mechanical and Manufacturing Engineering, Faculty of Engineering, University Putra Malaysia, \\ 43400 Serdang, Selangor, Malaysia
}

Correspondence should be addressed to Omid Boyer; omidboyer@gmail.com

Received 9 July 2013; Revised 9 November 2013; Accepted 18 November 2013

Academic Editor: Huijun Gao

Copyright (c) 2013 Omid Boyer et al. This is an open access article distributed under the Creative Commons Attribution License, which permits unrestricted use, distribution, and reproduction in any medium, provided the original work is properly cited.

\begin{abstract}
Technology progress is a cause of industrial hazardous wastes increasing in the whole world . Management of hazardous waste is a significant issue due to the imposed risk on environment and human life. This risk can be a result of location of undesirable facilities and also routing hazardous waste. In this paper a biobjective mixed integer programing model for location-routing industrial hazardous waste with two objectives is developed. First objective is total cost minimization including transportation cost, operation cost, initial investment cost, and cost saving from selling recycled waste. Second objective is minimization of transportation risk. Risk of population exposure within bandwidth along route is used to measure transportation risk. This model can help decision makers to locate treatment, recycling, and disposal centers simultaneously and also to route waste between these facilities considering risk and cost criteria. The results of the solved problem prove conflict between two objectives. Hence, it is possible to decrease the cost value by marginally increasing the transportation risk value and vice versa. A weighted sum method is utilized to combine two objectives function into one objective function. To solve the problem GAMS software with CPLEX solver is used. The problem is applied in Markazi province in Iran.
\end{abstract}

\section{Introduction}

The harmful wastes are produced from industries, manufacturers, hospitals, and other sectors which have harmful health effect on human and environment. The main sources of producing hazardous wastes are industrial and manufacturing processes; other generators can be household or commercial groups. There is wide and various range of industries to produce hazardous waste, for instance, chemicals manufacturing, oil refining, waste acids, and electroplating companies. Hazardous material is divided into four subgroups based on their features: toxicity (e.g., barium, cadmium, and chloroform); reactivity (e.g., sulfides and peroxide formers); corrosiveness (acids and bases); and ignitability (e.g., flammable liquid, oxidizers). These materials need specific transporting and treating to reduce their effect on environment and human health.
Definition of hazardous waste management is collecting, transporting, treating, recycling, and disposing waste residue under safe, efficient, and cost effective manner [1]. Two important objectives are included in transportation of hazardous waste: cost and risk. For carrier firms the best route is the shortest with minimum cost, but for government the basic problem is risk minimization. Hazardous waste also requires different technologies for treatment such as incineration, chemical, biological, and immobilization. Compatible treatment technology should be chosen based on waste features. In addition, recycling and disposing are two important stages in hazardous waste management.

The proposed framework for management of industrial hazardous waste is shown in Figure 1; it starts with generation nodes, and then wastes are divided into three groups. First group is recyclable waste; it is transferred to the recycling centers. Second group requires treatment facility, so they are 


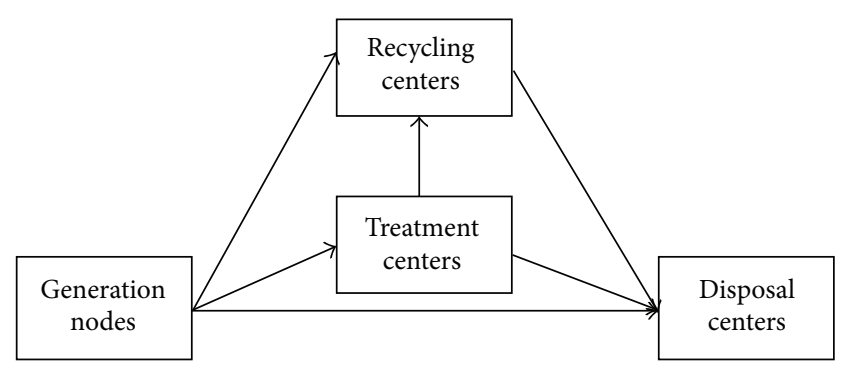

FIGURE 1: Proposed framework for hazardous waste management.

routed to the treatment centers with incineration technology. Third group is nonrecyclable and nontreatable waste, therefore it is transported to the disposal centers directly. In the end, residues from treatment and recycling centers are carried to the disposal centers.

At present, there have been little studies on the location of undesirable facilities and routing hazardous waste between these facilities simultaneously. So far, however, no research has been found that presented a comprehensive mathematical model based on proposed frame work (Figure 1). Recycling waste is an important stage in waste management [2], but this stage is often ignored in the literature. Therefore, in this model also locations for recycling centers are considered. In this study, a two-stage work is performed. First, appropriate site for undesirable facilities is selected by GIS. Second, the new mathematical model aids in locating facilities and routing waste. In addition, using total cost and transportation risk as objectives for this model can help decision makers to have a good tradeoff between environmental and economic aspects. In this way, using operational cost parameter for each facility and also cost saving parameter for recycled hazardous waste are suggested to have a more comprehensive cost objective. Applying the proposed model in a real case study showed that this model is practical in real world.

The remainder of this paper is arranged as follows. First a review from related paper is presented in Section 2. Mathematical model for proposed framework is formulated in Section 3. Section 4 gives a solution for multiobjective model. In Section 5, appropriate sites selection via GIS is illustrated and then the model is applied in Markazi province in Iran. A conclusion will be discussed in Section 6.

\section{Literature Review}

The initial works in the field of hazardous waste management only focused on locating facilities such as disposal and treatment centers. In this area a simple classification based on related papers considering hazardous waste management aspects will be illustrated as follows:

(1) locating undesirable facility,

(2) routing hazardous material via risk assessment,

(3) locating and routing hazardous material.

Several studies have addressed the location of undesirable facilities such as Ratick and White [3]. They provide a model with three objectives to locating undesirable facilities; the objectives are maximization of facility location cost, maximization of opposition, and equity maximization. Erkut and Neuman [4] also developed a multiobjective model which is the same as that of Ratick and White. They used maximizing of disutility objective instead of minimizing equity. In their model transportation cost is also considered. Wyman and Kuby [5] introduced a frame work and model for locating waste incineration with three conflicting objectives: minimizing cost, risk, and inequity. Rakas et al. [6] developed a multiobjective model to locate undesirable facilities with two objectives. First is to minimize cost including transportation and initial investment cost. Second objective is to minimize population opposition. They also believe that some input data in the model are not deterministic. Therefore, an extension of model has been proposed, considering uncertainty for a parameter. The fuzzy theory is used to show uncertainty for amount of produced waste per person. Locating incineration facilities is a sophisticated problem because of environmental and economic effects. Alçada-Almeida et al. [7] utilized a mixed integer multiobjective programming model to solve this problem. Their model has considered capacity for incineration. They also used a developed interactive decision support system by utilizing geographical information system (GIS) to select suitable option. Eiselt [8] worked on a model that is based on hub location model with cost minimization objective for locating landfills and transfer station. Krarup et al. [9] state the most locational models considering positive or negative effect for locating facilities close to demand center or far away from special places, respectively. They have presented a group of models with push and pull objectives for locating semiobnoxious facilities. In this way some papers have used different methods for locating undesirable facility such as Saameño et al. [10]. In their method, a weighted location problem in polygonal area is utilized.

Some models use assessment risk for routing and transporting hazardous material. Erkut and Verter [11] reviewed previous models to measure hazardous material transportation risk. They utilized one example to compare different types of risk models (traditional risk, population exposure, incident probability, conditional risk, and perceived risk). The results proved that various models have various consequences for choosing suitable transporting route. They believe selecting risk models for transporting HazMats (hazardous materials) requires more attention. Zhang et al. [12] applied Gaussian Plume model to model the number of people imposed by airborne contaminates. In this way, undesirable consequence probability is modeled to assess HazMats transportation as well as GIS to estimation risk. Erkut and Ingolfsson [13] believe that for satisfying risk models three reasonable axioms should be considered: path evaluating via monotonicity axiom, optimality principle for selecting path, and attribute monotonicity axiom. All previous models have violated from one or more axioms. They proposed new set models to fulfil all axioms. These axioms are also mentioned by Erkut and Verter [11]. Bonvicini and Spadoni [14] developed a new OPTIPATH model using TRAT4-GIS software to route HazMats and also decreasing risk. They illustrated that 
TRAT4-GIS is helpful for selecting suitable routes. Kazantzi et al. [15] proposed a framework for reducing risk and cost in transportation of hazardous material simultaneously. They used Mont Carlo simulation for finding impacts of risk incident and accident rate uncertainty on transportation model.

Several attempts have been made on locating and routing hazardous material concurrently. ReVelle et al. [16] developed mathematical model for locating storage facilities and selecting road for transporting spent fuel rods while minimizing transportation cost and perceived risk. Jacobs and Warmerdam [17] presented a biobjective model for hazardous waste locating and routing with risk and cost objective in 10month horizon plan. Their model considers hazardous waste risk in transporting, storage and disposal. Wyman and Kuby [5] have used a multiobjective mixed integer programming model for toxic waste. In their model a variable for choosing treatment technology is introduced and the goals of model are minimizing cost, risk and inequity. Giannikos [18] developed a multiobjective model for routing hazardous material and locating related facilities. A goal programming method has been used to solve the problem with four objectives including, operation cost minimization, perceived risk minimization, Minimum of Maximum individual perceived risk, and minimum of maximum disutility. Alumur and Kara [19] proposed a comprehensive new model for locating and routing hazardous waste with two objectives. First objective is to minimize total cost including transportation and fixed annual cost for opening a related facility. Second objective is to minimize transportation risk via population exposure method. In their model transporting different types of hazardous waste, compatibility of treatment technology with type of waste, percentage of recyclable waste, and percentage of mass reduction after using different treatment technology have been considered. Zhao [20] developed a locating and routing model for hazardous waste with the same objectives as those of Alumur and Kara [19] model. He used a goal programing method to solve the problem. In this area, Samanlioglu [2] also developed a multiobjective model based on $[19,20]$ models. Samanlioglu considered some additional features such as considering location for recycling centers, assessing site risk for hazardous waste facilities, and routing nonrecyclable waste from recycling centers to disposal centers. Xie et al. [21] proposed a multimodal hazardous material location-routing problem. In their model transfer yard location and suitable routes are optimized. Multiple modes in routing plans such as highways and railways are used.

Before proposing a model to locate facilities, finding suitable sites based on important criteria is necessary. One of the popular methods to select site in waste management is using GIS. Wang et al. [22] have used qualitative and quantitative factors to select proper site. Their proposed method has used the geographical information system (GIS) to select disposal site based on spatial data with environmental and economic criteria. Gorsevski et al. [23] have used multicriteria decision method based on geographical information system (GIS) for landfill site selection. Mahamid and Thawaba [24] also utilized (GIS) tool to find proper landfill site considering environmental factors. In this research, GIS tool will be utilized to find an appropriate site for treatment, recycling, and disposal centers based on some important criteria.

The complexity in hazardous waste management becomes apparent while solving model with conflict objectives simultaneously. In general one of the most popular approaches to solve such problems is combined multiobjectives to the single objective that is named the scalarization method. In this field the weighted sum method is more popular than other methods. Using the weighted sum method a group of supported efficient solutions from the Pareto frontier are achieved. Alumur and Kara [19] and Rakas et al. [6] are instances that have used this method in waste management. In this paper the weighted sum method will be utilized to find efficient solution from Pareto frontier.

The frame work of our model is close to the Alumur and Kara [19] and Samanlioglu [2]; they proposed a multiobjective model to location-routing hazardous waste. The difference between this paper and that of Alumur and Kara [19] included (1) establishing the locations for recycling centers and routing hazardous waste to these centers, (2) routing a part of generated hazardous waste to disposal centers directly, (3) considering population risk for all routes, (4) considering unit operation cost for treatment centers, disposal centers, and recycling centers in cost objective function, and (5) considering income from selling recycled waste in cost objective as cost saving parameter. Samanlioglu [2] also presented a comprehensive model with three objectives; our model is different from his model in some aspects and input data. We route a piece of generated hazardous waste to disposal centers directly. We take into account operation cost for all facilities and also recycled waste income in cost objective.

This assay attempts to develop a multiobjective model to locating-routing industrial hazardous waste with two objectives; for this reason the appropriate sites are selected to related facilities by GIS tool based on important criteria, then the weighted sum method is utilized to solve model and find efficient solution of Pareto frontier.

\section{Mathematical Model}

With respect to formulating location and routing problem for industrial hazardous waste, a multiobjective mixed integer programming model is proposed considering two conflicting objectives. First objective is minimization of total cost and second objective is minimization of transportation risk. The purpose of the model is to locate treatment centers with incineration technology and route hazardous waste to these centers, also to locate recycling centers and route hazardous waste from generation nodes and treatment centers to recycling centers, in addition, location of disposal centers and routing waste residue from other facilities to disposal centers.

Note that the model is an NP-hard problem; even single objective location routing problem is an NP-hard one [25]. A framework of model with input parameters and variables that help to understand the model easier is illustrated in Figure 2. The Objectives and constraints of this problem are as follows. 


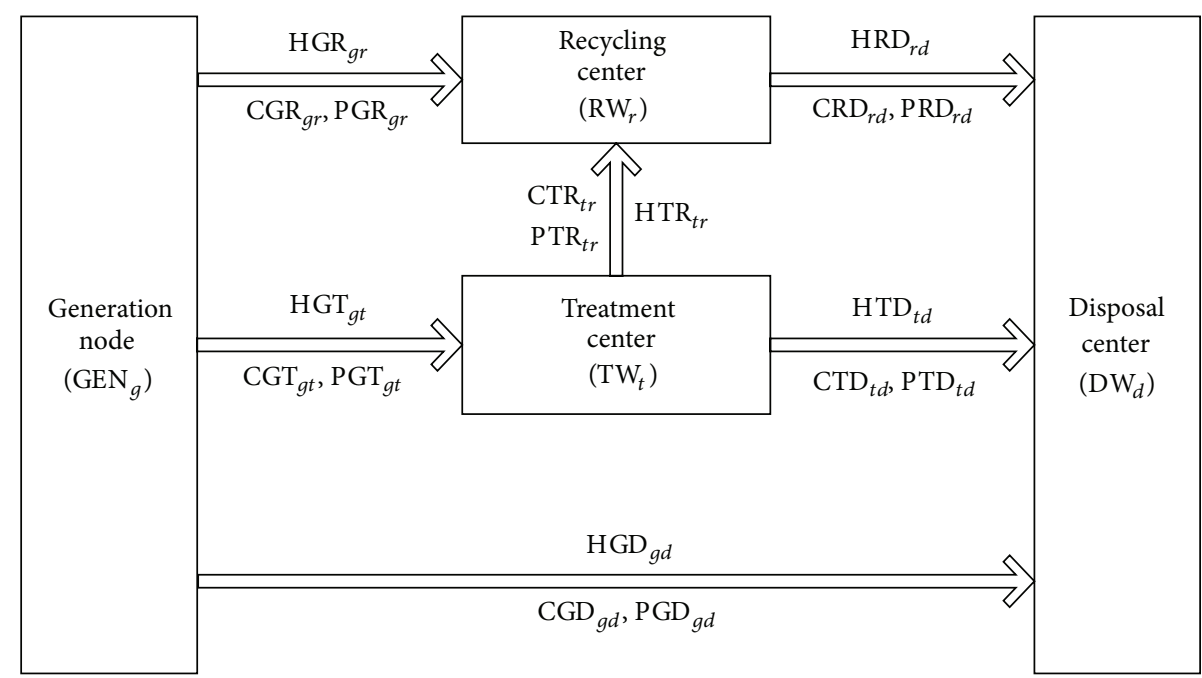

FIGURE 2: Input parameters and decision variables on proposed framework.

3.1. Objectives. Two objectives are considered in the model:

$$
\begin{gathered}
\operatorname{Minimize}\left(f_{1}\right)=\text { Total cost, } \\
\text { Minimize } f_{2}(x)=\text { Transportation risk. }
\end{gathered}
$$

3.1.1. Cost Objective. The total cost is formulated with sum of the transportation cost, operation cost, and initial investment cost to open facilities minus cost saving or income from recycled waste in recycling centers:

$$
\begin{aligned}
\operatorname{Minimize}_{1}(x)= & \sum_{g} \sum_{t} \mathrm{CGT}_{g t} * \mathrm{HGT}_{g t} \\
& +\sum_{g} \sum_{r} \mathrm{CGR}_{g r} * \mathrm{HGR}_{g r} \\
& +\sum_{g} \sum_{d} \mathrm{CGD}_{g d} * \mathrm{HGD}_{g d} \\
& +\sum_{t} \sum_{r} \mathrm{CTR}_{t r} * \mathrm{HTR}_{t r} \\
& +\sum_{r} \sum_{d} \mathrm{CRD}_{r d} * \mathrm{HRD}_{r d} \\
& +\sum_{t} \sum_{d} \mathrm{CT}_{t d} * \mathrm{HTD}_{t d} \\
& +\sum_{r} \mathrm{OCR}_{r} * \mathrm{RW}_{r}+\sum_{t} \mathrm{OCT}_{t} * \mathrm{TW}_{t} \\
& +\sum_{d} \mathrm{OCD}_{d} * \mathrm{DW}_{d}+\sum_{t} \mathrm{ICT}_{t} * Y_{t}
\end{aligned}
$$

$$
\begin{aligned}
& +\sum_{r} \mathrm{ICR}_{r} * X_{r}+\sum_{d} \mathrm{ICD}_{d} * Z_{d} \\
& -\sum_{r} \mathrm{IN}_{r} *\left(\mathrm{RW}_{r}-\sum_{d}\left(\mathrm{HRD}_{r d}\right)\right) .
\end{aligned}
$$

In this objective, transportation cost is calculated by multiplying unit transportation cost and amount of transported waste between two nodes. The operation cost is the cost of processing hazardous waste in treatment, recycling, and disposal centers that can be obtained by unit operation cost product to quantity of hazardous waste in each center. Initial investment cost is capital cost if a new facility is established for a node. Also, total cost saving or income from the recycled waste is estimated in the cost function.

3.1.2. Risk Objective. The transportation risk is considered for all routes between generation nodes, recycling centers, treatment centers, and disposal centers. To quantify risk, the exposure risk method is used. In this approach, the total number of people along a route during a hazardous waste transportation are considered. This objective considers environmental aspect to transport hazardous wastes and waste residues:

$$
\begin{aligned}
\text { Minimize } f_{2}(x)= & \sum_{g} \sum_{r} \mathrm{PGR}_{g r} * \mathrm{HGR}_{g r} \\
& +\sum_{g} \sum_{t} \mathrm{PGT}_{g t} * \mathrm{HGT}_{g t} \\
& +\sum_{g} \sum_{d} \mathrm{PGD}_{g d} * \mathrm{HGD}_{g d} \\
& +\sum_{r} \sum_{d} \mathrm{PRD}_{r d} * \mathrm{HRD}_{r d}
\end{aligned}
$$




$$
\begin{aligned}
& +\sum_{t} \sum_{d} \mathrm{PTD}_{t d} * \mathrm{HTD}_{t d} \\
& +\sum_{t} \sum_{r} \mathrm{PTR}_{t r} * \mathrm{HTR}_{t r} .
\end{aligned}
$$

3.2. Constraints. The model is under several reasonable constraints.

3.2.1. Mass Balance Constraint. There are flow balance constraints for each node of graph:

$$
\begin{gathered}
\mathrm{GEN}_{g}=\sum_{r} \mathrm{HGR}_{g r}+\sum_{t} \mathrm{HGT}_{g t}+\sum_{d} \mathrm{HGD}_{g d}, \quad \forall g \in G, \\
\sum_{r} \mathrm{HGR}_{g r}=f_{g} * \mathrm{GEN}_{g}, \quad \forall g \in G, \\
\mathrm{RW}_{r}=\sum_{g} \mathrm{HGR}_{g r}+\sum_{t} \mathrm{HTR}_{t r}, \quad \forall r \in R \\
\mathrm{TW}_{t}=\sum_{g} \mathrm{HGT}_{g t}, \quad \forall t \in T \\
\sum_{r} \mathrm{HTR}_{t r}=b_{t} * \mathrm{TW}_{t}, \quad \forall t \in T \\
\sum_{d} \mathrm{HGD}_{g d}=k_{g} * \mathrm{GEN}_{g}, \quad \forall g \in G, \\
\sum_{d} \mathrm{HRD}_{t d}=a_{t} * \mathrm{TW}_{t}-\sum_{r} \mathrm{HTR}_{t r}, \quad \forall t \in T \\
\sum_{d} \mathrm{HRD}_{r d}=\left(1-c_{r}\right) * \mathrm{RW}_{r}, \quad \forall r \in R, \\
\mathrm{DW}_{d}=\sum_{r} \mathrm{HRD}_{r d}+\sum_{t} \mathrm{HTD}_{t d}+\sum_{g} \mathrm{HGD}_{g d}, \quad \forall d \in D .
\end{gathered}
$$

Constraint (4) shows flow balance from generation nodes to other facilities. In this constraint, the amount of hazardous waste at generation nodes is equal to transported waste to recycling centers, treatment centers, and disposal centers. Constraint (5) says total amount of transported hazardous waste to the recycling centers has to be equal to the percentage of generated waste. Constraint (9) is also about transported hazardous waste from generation nodes to disposal centers. Constraints (6), (7), and (12) are amount of hazardous wastes that are recycled, treated and disposed in their centers, respectively. Constraint (8) denotes percentage of recyclable hazardous waste after treating. Constraints (10) and (11) present flow of hazardous waste between treatment centers and recycling centers to disposal centers, respectively.

3.2.2. Capacity Constraints. The capacity of facilities is limited to some factors such as equipment and manpower. Hence, a group of constraints are needed to display the maximum capacity of treatment, recycling, and disposal centers for a unit time. Constraints (13), (14), and (15) show this limitation. Also, to establish a new center minimum amount of hazardous waste or waste residue is required. Constraints (16), (17), and (18) are utilized as minimum capacity constraints for treatment, recycling and disposal centers, respectively:

$$
\begin{array}{cl}
\mathrm{RW}_{r} \leq \mathrm{CAR}_{r}^{u} X_{r}, & \forall r \in R, \\
\mathrm{TW}_{t} \leq \mathrm{CAT}_{t}^{u} Y_{t}, & \forall t \in T, \\
\mathrm{DW}_{d} \leq \mathrm{CAD}_{d}^{u} Z_{d}, & \forall d \in D, \\
\mathrm{RW}_{r} \geq \mathrm{CAR}_{r}^{l} X_{r}, & \forall r \in R, \\
\mathrm{TW}_{t} \geq \mathrm{CAT}_{t}^{l} Y_{t}, & \forall t \in T, \\
\mathrm{DW}_{d} \geq \mathrm{CAD}_{d}^{l} Z_{d}, & \forall d \in D .
\end{array}
$$

3.2.3. Variables Constraints. The problem is a mixed integer programming model; therefore, binary and nonnegative variables are utilized to formulate that. Constrain (19) is used for nonnegativity variables, and constraint (20) is considered for binary variables:

$$
\begin{aligned}
& \mathrm{HGR}_{g r} \geq 0, \forall g \in G, \quad \forall r \in R, \\
& \mathrm{HGT}_{g t} \geq 0, \forall g \in G, \quad \forall t \in T, \\
& \mathrm{HGD}_{g d} \geq 0, \quad \forall g \in G, \quad \forall d \in D, \\
& \mathrm{HTR}_{t r} \geq 0, \quad \forall t \in T, \quad \forall r \in R, \\
& \mathrm{HRD}_{r d} \geq 0, \quad \forall r \in R, \quad \forall d \in D, \\
& \mathrm{HTD}_{t d} \geq 0, \quad \forall t \in T, \quad \forall d \in D, \\
& \mathrm{TW}_{t} \geq 0, \quad \forall t \in T, \\
& \mathrm{RW}_{r} \geq 0, \quad \forall t \in T, \\
& \mathrm{DW}_{d} \geq 0, \quad \forall d \in D, \\
& Y_{t} \in\{0,1\}, \quad \forall t \in T, \\
& X_{r} \in\{0,1\}, \quad \forall r \in R, \\
& Z_{d} \in\{0,1\}, \quad \forall d \in D .
\end{aligned}
$$

If $g$ is the number of generation nodes, $t$ is the number of potential treatment nodes, $r$ is the number of potential recycling nodes, and $d$ is the number of potential disposal nodes, then the proposed model has $(r+t+d)$ binary variables and $(g r+g t+g d+t r+r d+t d+r+t+d)$ real decision variables. The number of total constraints of the proposed model without variable constraints is $(3 g+4 r+5 t+3 d)$.

\section{Proposed Solution to Multiobjective Problem}

Here, a multiobjective model with two conflict objectives was proposed. Multiobjective optimization [26] is to optimize two or more conflicting objectives with regard to a set of group 
constraints simultaneously. If the optimization of one objective leads to the automatic optimization of the other, it is not a multiobjective optimization. There is a group of solution methods for multiobjective problems called classical multiobjective optimization methods. To solve the model, the weighted sum method (WSM) is utilized. This method is a classical method that is used widely to solve multiobjective models. WSM combines two objectives to a single objective by multiplying each objective with a decision maker supplied weight. This method scalarizes the set of objectives with a convex combination. $f_{1}(x)$ and $f_{2}(x)$ have different units; after the objectives are normalized, a combined objective function can be created by summing the weighted normalized objectives and the multiobjective model is converted to a single objective model as follows [26]:

$$
\begin{gathered}
\operatorname{Min} f(x)=\frac{w_{1} * f_{1}(x)}{f_{1}^{*}(x)}+\frac{w_{2} * f_{2}(x)}{f_{2}^{*}(x)}, \\
w_{1}+w_{2}=1
\end{gathered}
$$

Here $w_{1}$ and $w_{2}$ are the weights of $f_{1}(x)$ and $f_{2}(x)$ objective function, respectively. First each objective is solved separately and optimal objectives are named $f_{1}^{*}(x)$ and $f_{2}^{*}(x)$. Thereafter $f_{1}(x) / f_{1}^{*}(x)$ and $f_{2}(x) / f_{2}^{*}(x)$ are weighted by $w_{1}$ and $w_{2}$; then two objectives are summed together. The single objective model is solved by considering different weights and constraints to have Pareto frontier of solutions. Amounts of obtained variables are inserted to the objectives to compute value of each objective function.

To have Pareto frontier of solutions the model is solved with different weights for each objective. There are two theorems in regard to classical method and also (21).

Theorem 1. The solution to the problem represented by (21) is Pareto-optimal if the weight is positive for all objectives.

Theorem 2. If $x^{*}$ is a Pareto-optimal solution of a convex multiobjective optimization problem, then there is a nonzero positive weight vector w such that $x^{*}$ is a solution to the problem in (21) (Figure 3).

\section{Analysis and Application for Province of Iran}

Analysis of model requires a real data; therefore, application of model is done in a province of Iran called Markazi. This province has 12 counties with a population of 1325159 and $29400 \mathrm{~km}^{2}$ area. There are fundamental industries in this province such as refinery, petrochemical industries, and aluminum manufacturers. There are 15 industrial areas in Markazi province with a total area of $14 \mathrm{~km}^{2}$. Amount of industrial hazardous waste in all counties is about 160000 ton per year. It is estimated to be around 700 tons per day considering 255 workdays in Iran. Out of 12 counties 7 are chosen for this model due to producing industrial hazardous waste. The information about the amount of hazardous waste in each county is not attainable. These amounts have been assumed proportional to industrial zone area in each county.

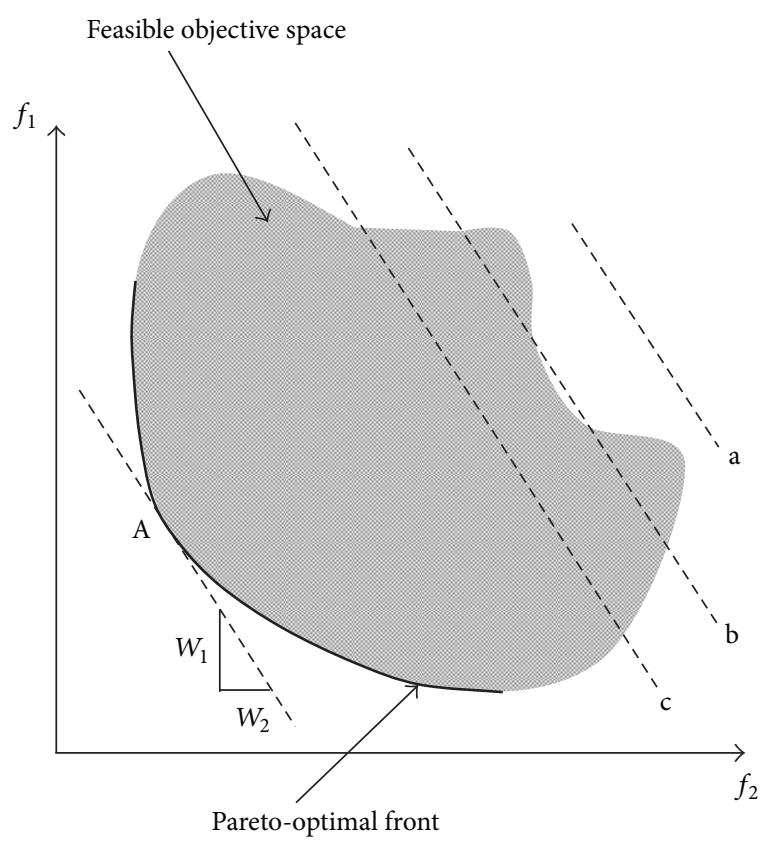

Figure 3: The weighted sum method on convex Pareto-optimal front.

For instance, the whole of industrial zone area in Arak county is $3 \mathrm{~km}^{2}$; hence the amount of produced industrial hazardous waste is 150 ton per day. The same method has been utilized for other industrial zones in different counties.

To select proper site for treatment, disposal, and recycling centers a geographical information system tool is applied. For GIS analysis Arc view (3.2) software has been used. Some input information is needed to carry out this software analysis. In this paper, common and significant criteria have been considered to select suitable area. These criteria are geology, topography, climate, land slope, distance from urban and rural area, protected area, infrastructure and distance to highway. Thereafter, analyzing with software, final site selection has been achieved. In a general manner all proper areas have been put in 2 groups: first is excellent area and second group is good area. These areas are illustrated in Figure 4. Based on amount of produced hazardous waste and capacity of facilities 5, 5, and 3 of selected areas are considered for treatment, disposal and recycling centers respectively. We tried to choose these 13 areas from excellent areas and also closer to big industrial zones. Selected areas are illustrated in Figure 5. To calculate transportation cost, fuel cost and amount of fuel that a truck consumes are considered. Average of the fuel consumption for a truck is about 0.5 liter per $\mathrm{km}$ in Iran, and price of fuel is $\$ 0.3$ per liter. Hence transportation cost is $\$ 0.15$ per $\mathrm{km}$, but in reality fuel cost is only 50 percent of trucks cost in transportation; therefore, it is around $\$ 0.3$ per $\mathrm{km}$. This cost is different for hazardous waste due to using specific equipment and trucks. Therefore, based on [2, 19] the unit transportation cost for $\mathrm{CTR}_{t r}, \mathrm{CDR}_{r d}$, and $\mathrm{CTD}_{t d}$ is $70 \%$ of hazardous waste. Capacity of these centers with regard to other centers in Iran is 100 tons, 250 tons, and 250 tons for recycling centers, treatment centers, and disposal centers, 


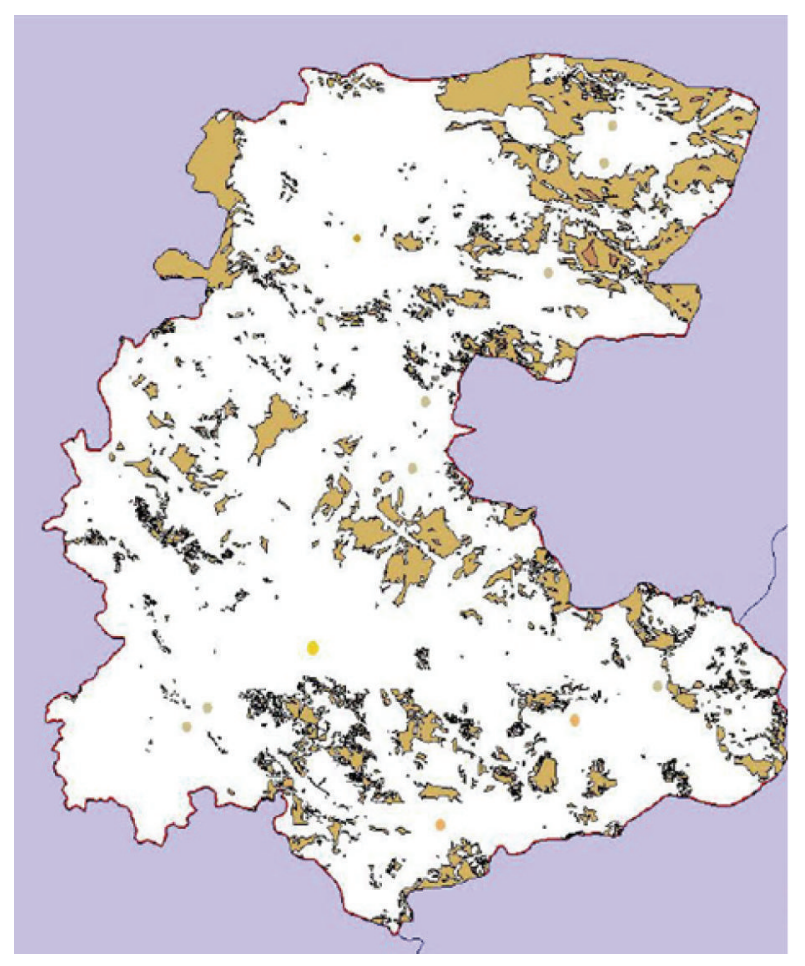

Final site selection

$\square$ Excellent

Good

FIGURE 4: Final site selection by GIS.

respectively. The investment cost to open a new center for hazardous waste based on previous price in Iran is around $\$ 10$ million for treatment centers, $\$ 5$ million for recycling center, and $\$ 7$ million for disposal centers. Operation costs in various centers are considered to be $\$ 200, \$ 250$, and $\$ 50$ per ton for treatment, recycling, and disposal centers, respectively. Income from recycled waste is about $\$ 700$. The population exposure bandwidth is considered to be $1000 \mathrm{~m}$ along the road here due the fact that ReVelle et al. [16] believed that different bandwidth does not have any effect on their solution. For this aim Arc GIS (3.2) has been used to estimate population in $1000 \mathrm{~m}$ bandwidth. In generation nodes $15 \%$ of generated hazardous waste is assumed recyclable and also $10 \%$ of generated hazardous waste is directly transported to disposal centers. Rate of mass reduction in incineration is about $80 \%$ based on $[2,19]$. In treatment centers $10 \%$ of treated waste is considered recyclable and is transported to recycling centers. Also in recycling centers $95 \%$ of hazardous waste is recycled and transported to the market or other factories.

Considering input data and problem framework, the model has been solved via GAMS software with CPLEX solver version 12.5 using a computer with Intel core i5$2.5 \mathrm{GHZ}, 4 \mathrm{~GB}$ memory.

To solve model different weights are utilized. Thus, with changing $w_{1}$ and $w_{2}$, also considering $w_{1}+w_{2}=1$, groups of weight vectors are produced to achieve efficient solution of

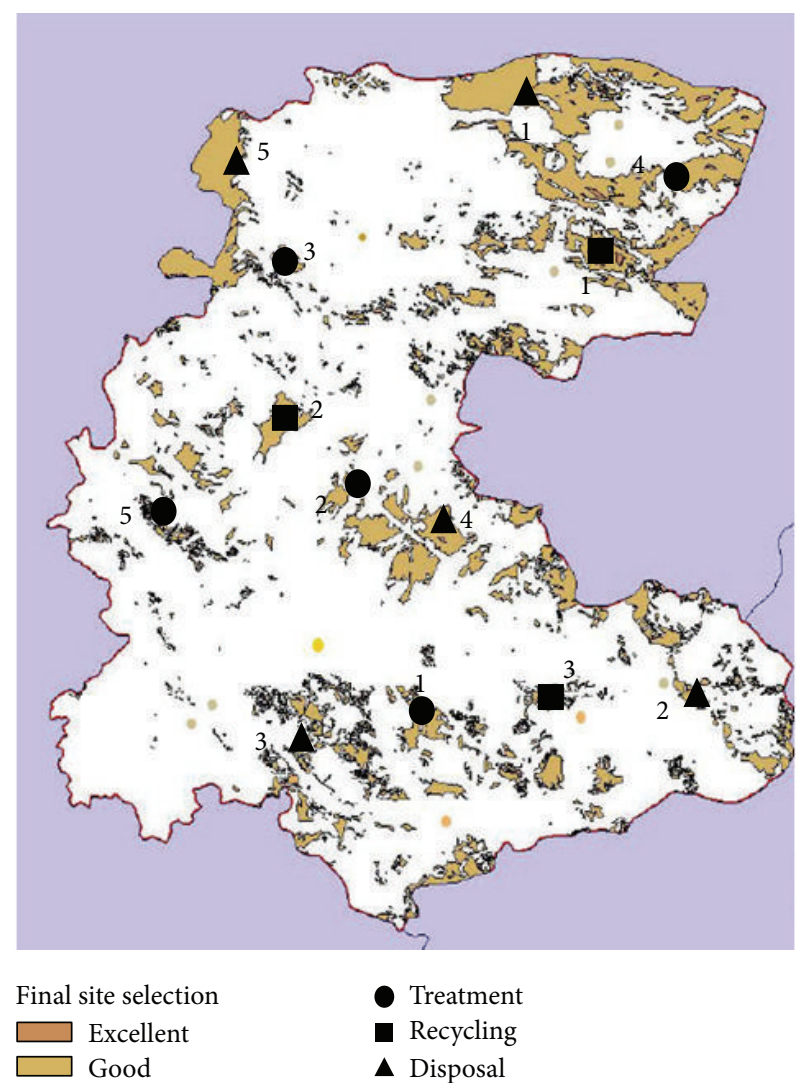

Figure 5: Appropriate site for waste management facilities.

the model from the Pareto set. In this paper 11 weight vectors have been used. In Table 1 weight vector groups, amount of each objective function based on its special weight, and generation time to solve model using GAMS software are illustrated. In Table 2, location of treatment centers, disposal centers and recycling centers with regard to 11 weight groups are presented.

The result in Table 1 illustrates a tradeoff between cost and risk objectives. The conflict between two objectives appeared by varying amount of $w_{1}$ and $w_{2}$ between 0 and 1 increments of 0.1 . It is clear that the cost value increases in order to decrease the risk value in Table 1 . The same result can be seen in Table 2. The location of treatment, recycling and disposal centers is changing by using different weight factors.

A sample of solved model with $w_{1}=0.5$ and $w_{2}=0.5$ is displayed in Figure 6; in this figure relation between chosen facilities is presented.

\section{Conclusion}

This assay has developed a multiobjective model to locatingrouting hazardous waste. This model is based on literature and studied models with some difference. First, considering a part of generated hazardous waste as nonrecyclable and nontreatable waste and routing it to disposal center directly. Second, to have more comprehensive model, operation cost for each facility and saving cost from selling recycled waste 
TABLE 1: Amount of two objective functions with regard to their weight vectors.

\begin{tabular}{|c|c|c|c|c|}
\hline Solution number & 1 & 2 & 3 & 4 \\
\hline$w_{1}$ & 0 & 0.1 & 0.2 & 0.3 \\
\hline$w_{2}$ & 1 & 0.9 & 0.8 & 0.7 \\
\hline$f_{1}(x)$ & $6.839707 * 10^{7}$ & $6.839707 * 10^{7}$ & $6.8397707 * 10^{7}$ & $6.442543 * 10^{7}$ \\
\hline$f_{2}(x)$ & 763254 & 763254 & 763254 & 849007 \\
\hline CPU time & 0.047 & 0.094 & 0.039 & 0.047 \\
\hline Solution number & 5 & 6 & 7 & 8 \\
\hline$w_{1}$ & 0.4 & 0.5 & 0.6 & 0.7 \\
\hline$w_{2}$ & 0.6 & 0.5 & 0.4 & 0.3 \\
\hline$f_{1}(x)$ & $6.442543 * 10^{7}$ & $6.342844 * 10^{7}$ & $6.342791 * 10^{7}$ & $6.342791 * 10^{7}$ \\
\hline$f_{2}(x)$ & 849007 & 864802 & 878519 & 878519 \\
\hline CPU time & 0.078 & 0.078 & 0.047 & 0.031 \\
\hline Solution number & 9 & 10 & 11 & \\
\hline$w_{1}$ & 0.8 & 0.9 & 1 & \\
\hline$w_{2}$ & 0.2 & 0.1 & 0 & \\
\hline$f_{1}(x)$ & $6.23202 * 10^{7}$ & $6161363 * 10^{7}$ & $6.136445 * 10^{7}$ & \\
\hline$f_{2}(x)$ & 895567 & 899487 & 977830 & \\
\hline CPU time & 0.036 & 0.093 & 0.062 & \\
\hline
\end{tabular}

TABLE 2: Location of different facilities considering various weight vectors.

\begin{tabular}{|c|c|c|c|c|}
\hline \multicolumn{2}{|c|}{ Weighs } & \multirow{2}{*}{$\begin{array}{c}\text { Treatment } \\
\text { centers }\end{array}$} & \multirow{2}{*}{$\begin{array}{l}\text { Disposal } \\
\text { centers }\end{array}$} & \multirow{2}{*}{$\begin{array}{l}\text { Recycling } \\
\text { centers }\end{array}$} \\
\hline$w_{1}$ & $w_{2}$ & & & \\
\hline 0 & 1 & $1,3,4$ & $1,2,3,5$ & 1,3 \\
\hline 0.1 & 0.9 & $1,3,4$ & $1,2,3,5$ & 1,3 \\
\hline 0.2 & 0.8 & $1,3,4$ & $1,2,3,5$ & 1,3 \\
\hline 0.3 & 0.7 & $1,3,4$ & $1,2,5$ & 1,3 \\
\hline 0.4 & 0.6 & $1,3,4$ & $1,2,5$ & 1,3 \\
\hline 0.5 & 0.5 & $2,3,4$ & $1,2,5$ & 1,3 \\
\hline 0.6 & 0.4 & $2,3,4$ & $1,2,5$ & 2,3 \\
\hline 0.7 & 0.3 & $2,3,4$ & $1,2,5$ & 2,3 \\
\hline 0.8 & 0.2 & $1,3,4$ & $1,3,5$ & 2,3 \\
\hline 0.9 & 0.1 & $1,3,4$ & $1,3,5$ & 1,2 \\
\hline 1 & 0 & $1,2,3$ & $1,3,4$ & 1,3 \\
\hline
\end{tabular}

were considered. The framework of this problem is location of treatment centers, recycling centers and disposal centers in suitable sites and routing industrial hazardous waste between them. Two conflict objectives have been considered. First, total cost minimization consists of transportation cost, operation cost, initial investment cost, and also cost saving parameter form earned income of selling recycled waste. Second objective is routing risk minimization using risk exposure method. To combine multiobjective model and the single objective model, the weighted sum method that is a kind of scalarization method was utilized. Application of model has been tested in Markazi province in Iran considering some assumptions for unavailable input data. The result of the solved example shows conflict between transportation

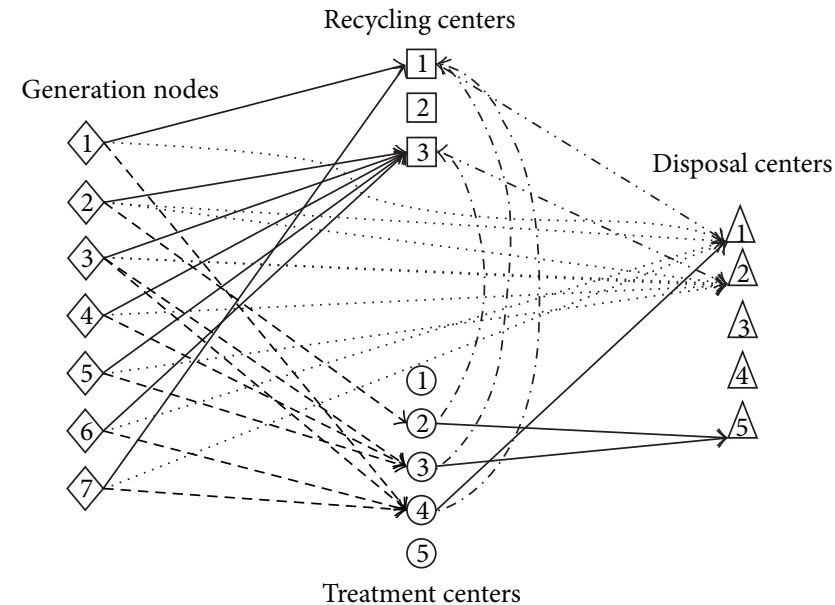

FIGURE 6: A sample of efficient solution for the model with equal weights.

risk and total cost objectives. For example, it is possible to decrease the cost objective with allocating higher weight (priority), while risk objective with lesser weight has a marginal increase. The same result can be taken in location of facilities. Their locations are changing when the weight vectors are varying. In this study, GAMS software with applying CPLEX solver has been used to solve problem.

The finding from this study suggests some future research. First, amount of hazardous waste depends on the number of industry and population and they vary in course of time. Hence, developing a model with long term planning for hazardous waste is necessary. Second, to solve multiobjective model in the field of locating-routing hazardous waste, 
scalarization method is common and popular. For such problem with conflicting criteria using metaheuristic method to encounter these problems with conflict objectives can be good as mentioned in Samanlioglu [2] and Alumur and Kara [19]. This study developed a new mathematical model based on a proposed framework; therefore, a data-driver framework [27] can be applied for future research. The last suggestion for future research is about amount of produced hazardous waste; in previous research amount of hazardous waste was considered deterministic, whereas amount of produced waste is uncertain in reality; hence fuzzy set theory can be useful in this way.

\section{Nomenclature}

Indices

G: Total of hazardous waste generation nodes $(1, \ldots, g)$

$T$ : Total of treatment center nodes $(1, \ldots, t)$

$R$ : Total of recycling center nodes $(1, \ldots, r)$

$D$ : Total of disposal center nodes $(1, \ldots, d)$.

Input Data

$\mathrm{CGT}_{g t}$ : Unit transportation cost from generation node $(g)$ to treatment center $(t)$

$\mathrm{CGR}_{g r}$ : Unit transportation cost from generation node $(g)$ to recycling center $(r)$

$\mathrm{CGD}_{g d}$ : Unit transportation cost from generation node $(g)$ to disposal center $(d)$

$\mathrm{CTD}_{t d}$ : Unit transportation cost from treatment center $(t)$ to disposal center $(d)$

$\mathrm{CTR}_{t r}$ : Unit transportation cost from treatment center $(t)$ to recycling center $(r)$

$\mathrm{CRD}_{r d}$ : Unit transportation cost from recycling center $(r)$ to disposal center $(d)$

$\mathrm{OCT}_{t}$ : Unit operation cost at treatment center $(t)$

$\mathrm{OCR}_{r}$ : Unit operation cost at recycling center $(r)$

$\mathrm{OCD}_{d}$ : Unit operation cost at disposal center $(d)$

$\mathrm{ICT}_{t}$ : Initial investment cost to open a new treatment center $(t)$

$\mathrm{ICR}_{r}$ : Initial investment cost to open a new recycling center $(r)$

$\mathrm{ICD}_{d}$ : Initial investment cost to open a new disposal center $(d)$

$\mathrm{IN}_{r}$ : Unit income from selling recycled hazardous waste $(r)$

$\mathrm{PGR}_{g r}$ : Population within bandwidth along route from generation node $(g)$ and recycling center $(r)$

$\mathrm{PGT}_{g t}$ : Population within bandwidth along route from generation node $(g)$ and treatment center $(t)$

$\mathrm{PGD}_{g d}$ : Population within bandwidth along route from generation node $(g)$ and disposal center $(d)$
$\mathrm{PTR}_{t r}$ : Population within bandwidth along route from treatment center $(t)$ and recycling center $(r)$

$\mathrm{PRD}_{r d}$ : Population within bandwidth along route from recycling center $(r)$ and disposal center (d)

$\mathrm{GEN}_{g}$ : Quantity of produced hazardous waste at generation nodes $(g)$

$a_{t}$ : $\quad$ Percentage of hazardous waste mass reduction after treating at treatment centers $(t)$

$b_{t}$ : $\quad$ Percentage of recyclable hazardous waste after treating at treatment centers $(t)$

$c_{r}$ : Percentage of recycled hazardous waste at recycling centers $(r)$

$f_{g}$ : Percentage of recyclable hazardous waste generated at generation nodes $(g)$

$k_{g}$ : $\quad$ Percentage of disposable hazardous waste generated at generation nodes $(g)$

$\mathrm{CAR}_{r}^{u}$ : Maximum capacity of recycling center $(r)$

$\mathrm{CAT}_{t}^{u}$ : Maximum capacity of treatment center $(t)$

$\mathrm{CAD}_{d}^{u}$ : Maximum capacity of disposal center $(d)$

$\mathrm{CAR}_{r}^{l}$ : Minimum capacity of recycling center $(r)$

$\mathrm{CAT}_{t}^{l}$ : Minimum capacity of treatment center $(t)$

$\mathrm{CAD}_{d}^{l}$ : Minimum capacity of disposal center $(d)$.

Variables

$\mathrm{HGR}_{g r}$ : Transported hazardous waste quantity from generation nodes $(g)$ to recycling center $(r)$

$\mathrm{HGT}_{g t}$ : Transported hazardous waste quantity from generation nodes $(g)$ to treatment center $(t)$

$\mathrm{HGD}_{g d}$ : Transported hazardous waste quantity from generation nodes $(g)$ to disposal center $(r)$

$\mathrm{HTR}_{t r}$ : Transported hazardous waste quantity from treatment center $(t)$ to recycling center $(r)$

$\mathrm{HRD}_{r d}$ : Transported hazardous waste quantity from recycling center $(r)$ to disposal center $(d)$

$\mathrm{HTD}_{t d}$ : Transported hazardous waste quantity from treatment center $(t)$ to recycling center $(d)$

$\mathrm{RW}_{r}$ : Amount of hazardous waste recycled at recycling center $(r)$

$\mathrm{TW}_{t}$ : Amount of hazardous waste treated at treatment center $(t)$

$\mathrm{DW}_{d}$ : Amount of hazardous waste disposed at disposal center $(d)$

$X_{r}$ : $\quad$ Binary variable: 1 if recycling center establishes to node $(r), 0$ otherwise

$Y_{t}: \quad$ Binary variable: 1 if treatment center establishes to node $(t), 0$ otherwise

$Z_{d}$ : $\quad$ Binary variable: 1 if disposal center establishes to node $(d), 0$ otherwise.

\section{References}

[1] A. K. Nema and S. Gupta, "Optimization of regional hazardous waste management systems: an improved formulation," Waste Management, vol. 19, no. 7, pp. 441-451, 1999. 
[2] F. Samanlioglu, "A multi-objective mathematical model for the industrial hazardous waste location-routing problem," European Journal of Operational Research, vol. 226, no. 2, pp. 332340, 2013.

[3] S. J. Ratick and A. L. White, "A risk-sharing model for locating noxious facilities," Environment \& Planning B, vol. 15, no. 2, pp. 165-179, 1988.

[4] E. Erkut and S. Neuman, "A multiobjective model for locating undesirable facilities," Annals of Operations Research, vol. 40, no. 1, pp. 209-227, 1992.

[5] M. M. Wyman and M. Kuby, "Proactive optimization of toxic waste transportation, location and technology," Location Science, vol. 3, no. 3, pp. 167-185, 1995.

[6] J. Rakas, D. Teodorović, and T. Kim, "Multi-objective modeling for determining location of undesirable facilities," Transportation Research Part D, vol. 9, no. 2, pp. 125-138, 2004.

[7] L. Alçada-Almeida, J. Coutinho-Rodrigues, and J. Current, "A multiobjective modeling approach to locating incinerators," Socio-Economic Planning Sciences, vol. 43, no. 2, pp. 111-120, 2009.

[8] H. A. Eiselt, "Locating landfills-Optimization vs. reality," European Journal of Operational Research, vol. 179, no. 3, pp. 10401049, 2007.

[9] J. Krarup, D. Pisinger, and F. Plastria, "Discrete location problems with push-pull objectives," Discrete Applied Mathematics, vol. 123, no. 1-3, pp. 363-378, 2002.

[10] J. J. Saameño Rodríguez, C. Guerrero García, J. Muñoz Pérez, and E. Mérida Casermeiro, "A general model for the undesirable single facility location problem," Operations Research Letters, vol. 34, no. 4, pp. 427-436, 2006.

[11] E. Erkut and V. Verter, "Modeling of transport risk for hazardous materials," Operations Research, vol. 46, no. 5, pp. 625-642, 1998.

[12] J. Zhang, J. Hodgson, and E. Erkut, "Using GIS to assess the risks of hazardous materials transport in networks," European Journal of Operational Research, vol. 121, no. 2, pp. 316-329, 2000.

[13] E. Erkut and A. Ingolfsson, "Transport risk models for hazardous materials: revisited," Operations Research Letters, vol. 33, no. 1, pp. 81-89, 2005.

[14] S. Bonvicini and G. Spadoni, "A hazmat multi-commodity routing model satisfying risk criteria: a case study," Journal of Loss Prevention in the Process Industries, vol. 21, no. 4, pp. 345358, 2008.

[15] V. Kazantzi, N. Kazantzis, and V. C. Gerogiannis, "Risk informed optimization of a hazardous material multi-periodic transportation model," Journal of Loss Prevention in the Process Industries, vol. 24, no. 6, pp. 767-773, 2011.

[16] C. ReVelle, J. Cohon, and D. Shobrys, "Simultaneous siting and routing in the disposal of hazardous wastes," Transportation Science, vol. 25, no. 2, pp. 138-145, 1991.

[17] T. L. Jacobs and J. M. Warmerdam, "Simultaneous routing and siting for hazardous-waste operations," Journal of Urban Planning \& Development, vol. 120, no. 3, pp. 115-131, 1994.

[18] I. Giannikos, "A multiobjective programming model for locating treatment sites and routing hazardous wastes," European Journal of Operational Research, vol. 104, no. 2, pp. 333-342, 1998.

[19] S. Alumur and B. Y. Kara, "A new model for the hazardous waste location-routing problem," Computers and Operations Research, vol. 34, no. 5, pp. 1406-1423, 2007.
[20] J. Zhao, "Model and algorithm for hazardous waste locationrouting problem," in Proceedings of the International Conference of Logistics Engineering and Management (ICLEM '10), pp. 2843-2849, 2010.

[21] Y. Xie, W. Lu, W. Wang et al., "A multimodal location and routing model for hazardous materials transportation," Journal of Hazardous Materials, vol. 227, pp. 135-141, 2012.

[22] G. Wang, L. Qin, G. Li, and L. Chen, "Landfill site selection using spatial information technologies and AHP: a case study in Beijing, China," Journal of Environmental Management, vol. 90, no. 8, pp. 2414-2421, 2009.

[23] P. V. Gorsevski, K. R. Donevska, C. D. Mitrovski, and J. P. Frizado, "Integrating multi-criteria evaluation techniques with geographic information systems for landfill site selection: a case study using ordered weighted average," Waste Management, vol. 32, no. 2, pp. 287-296, 2012.

[24] I. Mahamid and S. Thawaba, "Multi criteria and landfill site selection using GIS: a case study from Palestine," Open Environmental Engineering Journal, vol. 3, pp. 33-41, 2010.

[25] G. Nagy and S. Salhi, "Location-routing: issues, models and methods," European Journal of Operational Research, vol. 177, no. 2, pp. 649-672, 2007.

[26] K. Deb, "Multi-objective optimization," in Multi-Objective Optimization Using Evolutionary Algorithms, pp. 13-46, 2001.

[27] S. Yin, S. X. Ding, A. Haghani et al., "A comparison study of basic data-driven fault diagnosis and process monitoring methods on the benchmark Tennessee Eastman process," Journal of Process Control, vol. 22, no. 9, pp. 1567-1581, 2012. 


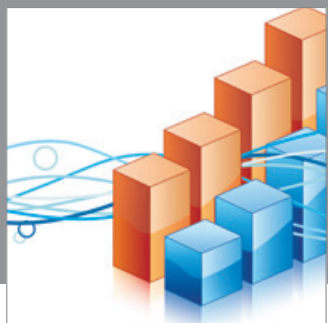

Advances in

Operations Research

mansans

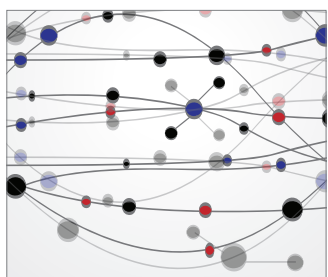

The Scientific World Journal
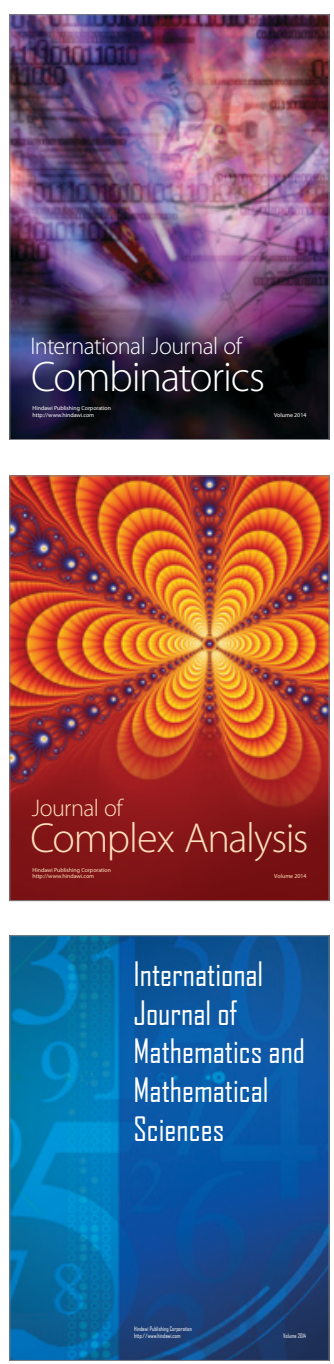
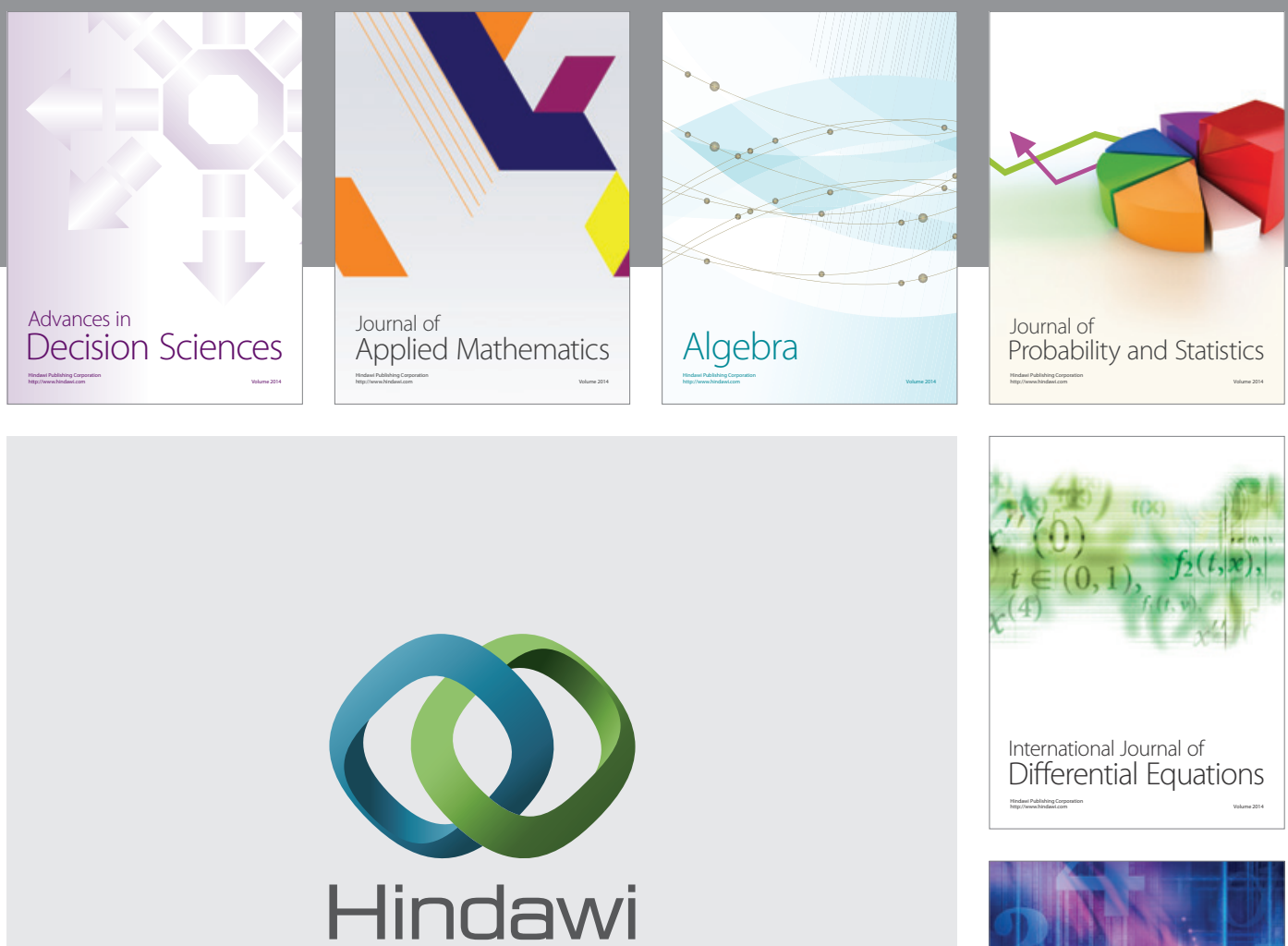

Submit your manuscripts at http://www.hindawi.com
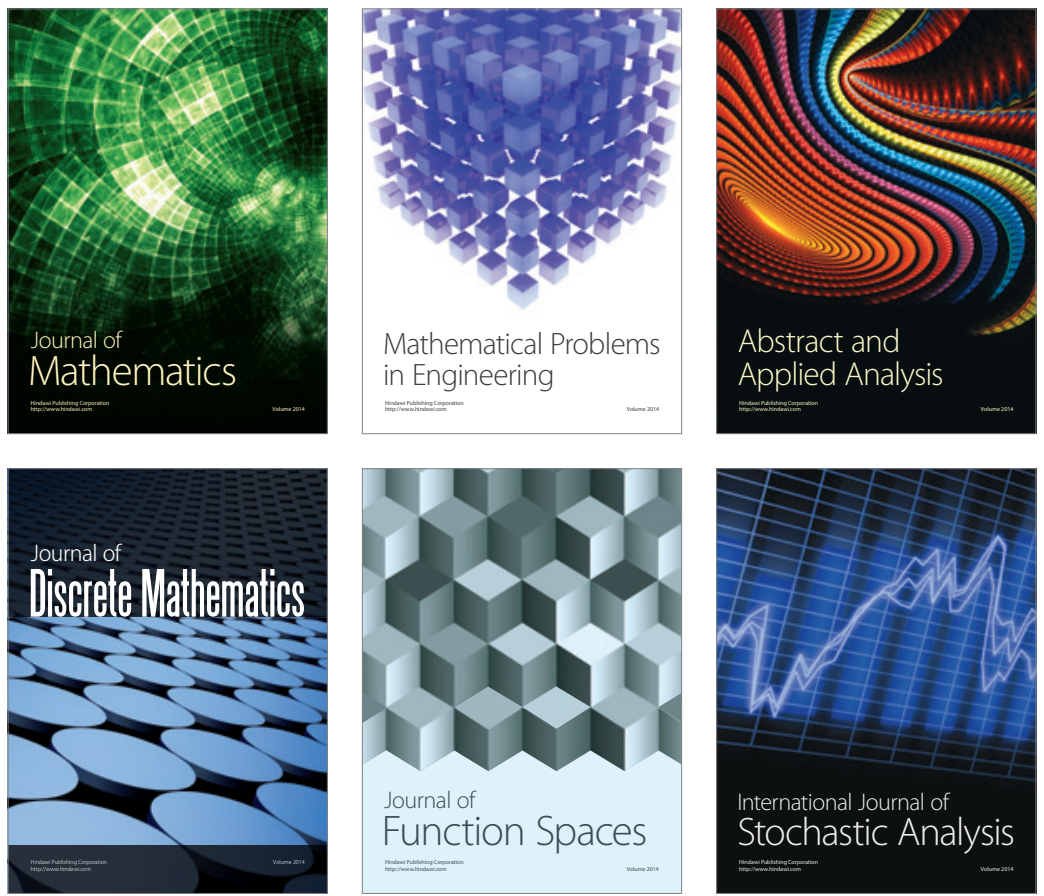

Journal of

Function Spaces

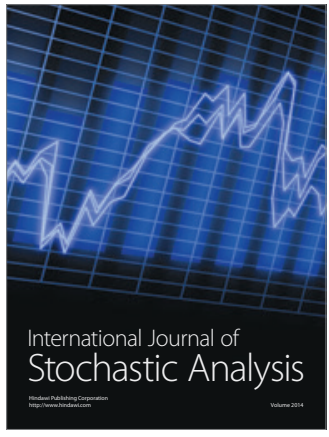

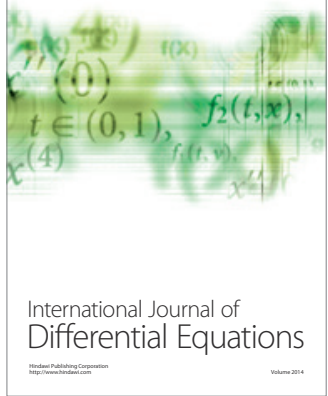
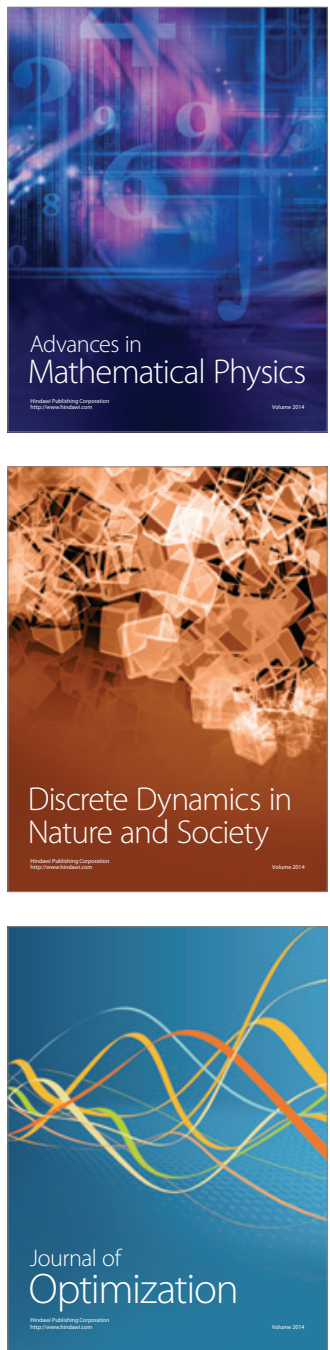